Informations

Orthop Traumatol (1994) $4: 16$

\title{
Avril 1994
}

\section{4ème Réunion annuelle du}

\section{Groupe d'Etude des Tumeurs Osseuses}

Nice, 25-26 mars 1994

Renseignements : Pr C. Argenson, Service d'Orthopédie-Traumatologie, Hôpital St Roch, BP 319, 06006 Nice Cedex 1, Tél. 92.03.33.38, Fax 92.03.33.52

\section{Mai 1994}

\section{Cours de perfectionnement en anatomie chirurgicale et techniques chirurgicales du rachis}

\section{Marseille, 16-20 mai 1994}

organisé par Pr René Louis

Thèmes : - Anatomic chirurgicale, abords antérieurs et postérieurs, osthéosynthèses par plaques vissées

- Techniques chirurgicales et indications : spondylolyses, spondylolisthesis, arthroses, hernies discales, tumeurs, traumatismes et malformations

- Travaux pratiques sur sujets anatomiques

- Audio-visuel (22 films)

Langue officielle : français

Renseignements : Pr René Louis, Cours sur le rachis, Hôpital de la Conception, 147, bd Baille, 13005 Marseille, Tél. 91.38.37.28, Fax 91.47.24.38

Inscriptions : M.C.O. Congrès, Le Pullman, 255, avenue du Prado, 13008 Marseille, Tél. 91.78.90.74, Fax 91.78.90.71

\section{AIOD \\ Association Internationale pour l'Ostéosynthèse Dynamique}

Jubilé des 20 ans du clou verrouillé Cours de traumatologie

\section{Strasbourg, 18-22 avril 1994}

Renseignements : Association Internationale pour l'Ostéosynthèse Dynamique, 3, rue du Général Offenstein, 67100 Strasbourg, Tél. 88.39.62.90, Télex (042) 871098 F, Fax 88.39.43.96

\section{European Congress on Bone and Joint Infections}

Leuven, Belgium, May 13-14, 1994

Organized by the European Bone and Joint Infection Society

Main topics:

- Diabetic foot infections

- Sampling of bacteriological specimens

- Post-operative infections after spine instrumention

- Free papers

Scientific Secretariat: Dr J. Stuyck (Chairman), Department of Orthopaedics, University Hospital, Weligerveld 1, B-3212 Pellenberg, Belgium 